\title{
Patterns of Consultation-liaison Psychiatry among Inpatients at a Tertiary Care Hospital
}

\author{
Darpan Kaur ${ }^{1}$, Rishab Verma ${ }^{2}$, Shaunak Ajinkya ${ }^{3}$, Rakesh Ghildiyal ${ }^{4}$
}

\begin{abstract}
Background: There is sparse literature on the profile of consultation-liaison psychiatry (CLP) across tertiary institutions in developing countries like India.

Aims: To assess specific patterns of referrals to psychiatry, psychiatric diagnosis, and interventions among inpatients admitted at a tertiary care hospital and to explore demographic and correlation factors.

Materials and methods: An observational type of cross-sectional study was conducted assessing consecutive inpatients fulfilling the inclusion and exclusion criteria referred to the Psychiatry Department for Consultation-liaison Psychiatry. Institutional Ethics Committee (IEC) clearance was obtained. A structured predesigned data collection form was used to assess relevant variables, and analysis was done comprising frequency analysis, mean, percentage, chi-square test, analysis of variance (ANOVA), and multiple logistic regressions.

Results: We analyzed a convenient sample profile of 100 referrals. The mean age of the sample was 40.95 years $(S D=18.05)$ with $70 \%$ males. Eighty-one percent were from rural areas with $19 \%$ from urban areas. The mean days of referral were 2.27 days (SD $=3.155)$. The medicine department contributed to maximum referrals (60\%). Common reasons for referral were substance use (23\%) and self-harm (20\%). The most common psychiatric diagnosis was alcohol dependence (24\%). The dual diagnosis was observed in $10 \%$ of patients. Correlational regression analysis found that alcohol dependence was significantly seen in males, while depression and adjustment disorders were seen in females. Fiftyeight percent received oral psychiatric medications, while $42 \%$ received only psychotherapy. Seventy-six percent of patients/caregivers were not adequately communicated by the referring team about specific reasons for reference to psychiatry.

Conclusion: Our study has relevant implications for research and clinical care across CLP.

Keywords: Consultation-liaison psychiatry, Inpatients, Patterns, Referral, Tertiary care hospital.

Indian Journal of Private Psychiatry (2020): 10.5005/jp-journals-10067-0052
\end{abstract}

\section{INTRODUCTION}

Consultation-liaison psychiatry (CLP) is the study, practice, and teaching of the relation between medical and psychiatric disorders. ${ }^{1}$ It is a subspecialty of psychiatry that provides care for inpatients under non-psychiatric care. ${ }^{2}$ Background literature has assessed factors, such as referral rates to psychiatry, reasons for referral, specialties, sociodemographic factors, utilization of psychiatric services, communication regarding referral, and intervention patterns. ${ }^{3-6}$ Consultation-liaison psychiatry is described to be one of the young and upcoming subspecialties of psychiatry in India. ${ }^{7}$ It is important to prioritize and understand the perceived need for improvement in the existing CLP services across the country and enhance training and research work. A national collaborative survey on consultation-liaison services conducted across multiple institutions in India found that delirium emerged as the topmost common psychiatric diagnosis among $79(87.8 \%)$ institutes. It was further followed by substance use disorders (70\%), self-harm (60\%), and depression (38.9\%) as the most common psychiatric diagnosis seen across CLP study settings. ${ }^{8}$

A study done by Patra et al. found that the emergency department contributed to the majority of referrals across CLP in their study settings. ${ }^{9}$ Grover et al. found that delirium emerged as the most common diagnosis among referrals made to the CLP team. ${ }^{10}$ Another CLP-based study conducted by Hashim et al. found that the significant pattern of referrals across their study settings was associated with a relatively younger age, positive past and family history of suicidal behavior, mode of attempt, and a longer duration of hospitalization. ${ }^{11}$ It is recommended
1,2,4 Department of Psychiatry, Mahatma Gandhi Missions Medical College and Hospital, Navi Mumbai, Maharashtra, India

${ }^{3}$ Consultant Psychiatrist, Kokilaben Dhirubhai Ambani Hospital and Medical Research Institute, Andheri, Mumbai, Maharashtra, India.

Corresponding Author: Darpan Kaur, Associate Professor of Psychiatry, Department of Psychiatry, Mahatma Gandhi Missions Medical College and Hospital, Navi Mumbai, Maharashtra, India, Phone: +91 9769634045, e-mail: kaur.darpan@gmail.com

How to cite this article: Kaur D, Verma R, Ajinkya S, et al. Patterns of Consultation-liaison Psychiatry among Inpatients at a Tertiary Care Hospital. Ind J Priv Psychiatry 2020;14(1):35-40.

Source of support: Nil

Conflict of interest: None

that studies conducted on referral patterns and modes of clinical presentation across CLP are relevant and useful for planning and organizing these services across the general and specialist centers. $^{12}$

\section{Aims and Objectives}

The aim of the study was to assess patterns of referral for CLP among inpatients admitted at a tertiary care hospital. The objectives of the study were to assess distinct patterns of referral in terms of demographic factors, psychiatric and medical comorbidity, psychiatric intervention, and communication regarding referral and explore significant correlations among the same. 


\section{Subject and Methods}

The study was an observational type of cross-sectional survey conducted at a tertiary care hospital. Consecutive inpatients referred for CLP opinion and fulfilling the inclusion and exclusion criteria were assessed and comprised the sample for the study. The inclusion criterion was that the patients should be above 18 years of age. Patients with severe medical or psychiatric morbidities rendering them uncooperative to give consent were excluded from the study. A predesigned data collection form was used to assess the following variables comprising of demographic factors of age, sex, and residence, common reasons for referral to psychiatry unit, referring units, period of referral to psychiatry since the time of admission in the referring unit and communication regarding psychiatric referral to patients/ caregivers, medical comorbidity, comorbid substance use, family history of psychiatric disorders, psychiatric comorbidity, and psychiatric interventions. Institutional Ethics Committee (IEC) clearance was obtained. Informed consent was obtained for the study. A convenient sample size of 100 was analyzed with appropriate statistics comprising descriptive tests, chi-square tests, and regression analysis.

\section{Results}

The mean age of our sample was 40.95 years $(S D=18.05)$ with a minimum age of 18 years and a maximum of 95 years. Seventy percent were males and 30\% females. Eighty-one percent were hailing from rural areas and the rest from urban areas (Table 1). The mean days of referral to psychiatry were 2.27 days $(S D=3.155)$ with the earliest referral being done on day one and the latest being 14 days after the time of admission. The maximum numbers of referrals were done on day one of admission (36\%). There was a downtrend

Table 1: Sociodemographic characteristics $(n=100)$

\begin{tabular}{ll}
\hline Demographic and clinical factors & Percentage $(n=100)$ \\
\hline Gender & $70 \%$ \\
Male & $30 \%$ \\
Female & \\
Residence & $81 \%$ \\
Rural area & $19 \%$ \\
Urban area & \\
Reasons for referral & $23 \%$ \\
Substance use & $20 \%$ \\
Self-harm & $15 \%$ \\
Known case of psychiatric illness & $14 \%$ \\
Counseling & $10 \%$ \\
Irrelevant talking & $8 \%$ \\
Patient appearing sad/tensed & $6 \%$ \\
Unexplained medical symptoms & $4 \%$ \\
Patient being uncooperative/rowdy & \\
Family history of psychiatric diagnosis & $11 \%$ \\
Present & $89 \%$ \\
Absent & \\
Dual psychiatric diagnosis & $10 \%$ \\
Present & $90 \%$ \\
Absent &
\end{tabular}

observed after that with a $1 \%$ referral rate from day 11 to day 14 after admission as shown in Figure 1. The medicine department contributed to maximum referrals (60\%) (Fig. 2). Other referring departments were surgery (25\%), orthopedics (8\%), ENT (2\%), obstetrics (2\%), casualty (2\%), and ophthalmology (1\%).

The common reasons for referral were substance use (23\%) and self-harm (20\%). The other reasons for reference were patient being a known case of psychiatric illness (15\%), counseling (14\%), irrelevant talking (10\%), patient appearing sad/tensed (8\%), unexplained medical symptoms (6\%), and patient being uncooperative/rowdy (4\%). Cases of poisoning (17\%) were the most commonly referred cases to psychiatry from the medicine department, infections were the most common referrals from surgical patients, and fractures were the most common orthopedics diagnosis in the referred sample. Organophosphates poisoning was the most common form of poisoning amounting to $8 \%$ of cases. The most common psychiatric diagnosis was alcohol dependence (24\%) (Fig. 3). The International Classification of Diseases (ICD-10) diagnostic profile as per broad categories was substance dependence (27\%), organic mental conditions (18\%), schizophrenia and other psychosis (7\%),

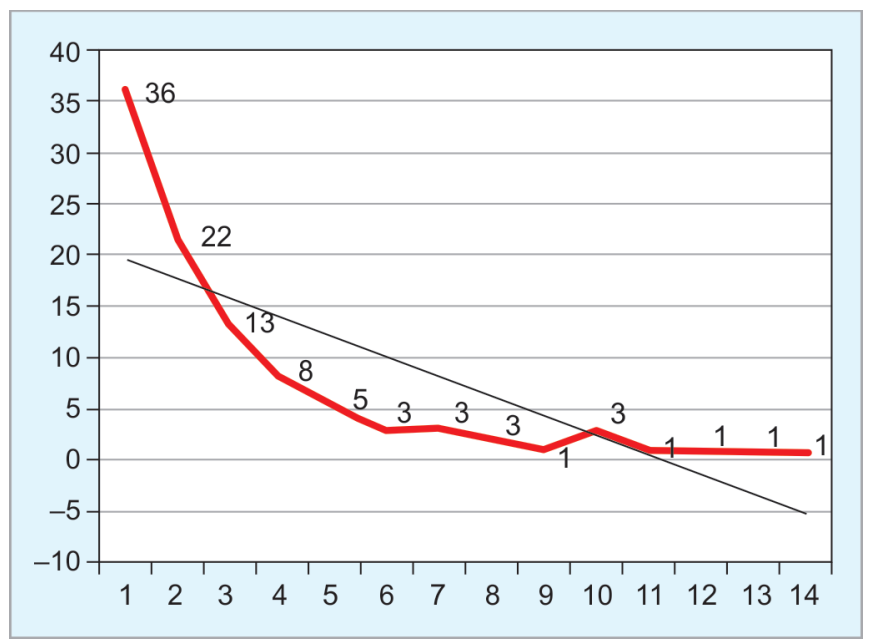

Fig. 1: Days of referral to CLP since the time of admission

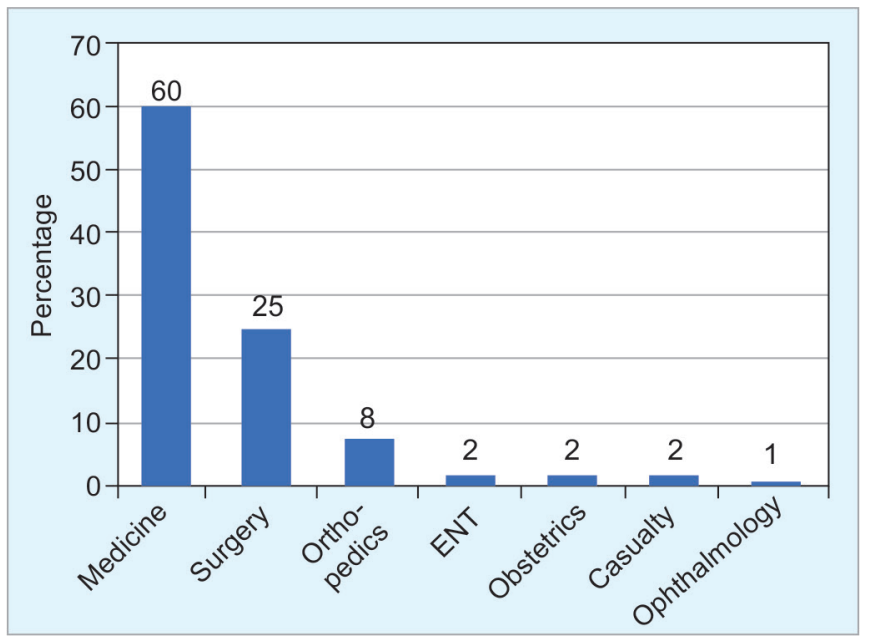

Fig. 2: Profile of referring departments for CLP 


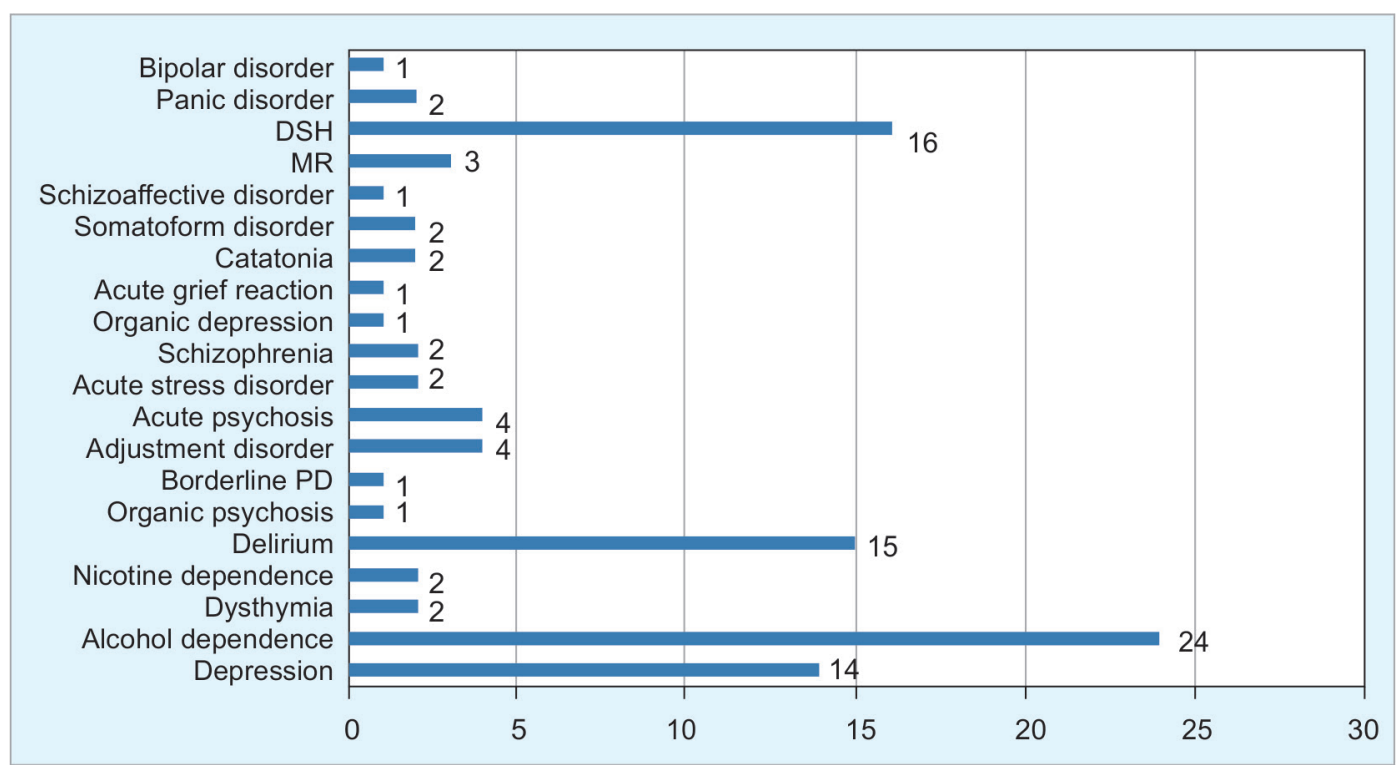

Fig. 3: Profile of psychiatric diagnosis (ICD-10) for the sample $(n=100)$

mood disorders (17\%), anxiety and stress-related disorders (10\%), mental retardation (3\%), and Z category of deliberate self-harm (18\%). The dual psychiatric diagnosis was found in $10 \%$. A positive family history of psychiatric illness was found in $11 \%$ of cases.

Seventy-four percent of the patients and the caregivers were not fully communicated and explained the reason for referral by their referring doctor despite written reference to psychiatry. Regarding the psychiatric interventions received by the referred patients, $58 \%$ received oral psychiatric medications while $42 \%$ received only psychotherapy. Thirty percent of patients received vitamins, and $29 \%$ received injectable antipsychotic medications. Antipsychotics and benzodiazepines together were most commonly prescribed (14\%) with antipsychotics alone amounting to $12 \%$ and antidepressants alone amounting to $12 \%$.

The following variables emerged to be significant on chisquare and regression analysis. The referring unit emerged to eb significant with the psychiatric diagnosis $(p<0.001)$ and ICD-10 diagnosis $(p<0.001)$. Gender was found to be significantly associated with psychiatric diagnosis $(p<0.003)$, ICD-10 diagnosis $(p<0.006)$, and referring unit $(p<0.048)$. The variable psychiatric diagnosis had a significant association with oral psychopharmacological medications ( $p<0.001$ ). It was further found that the variable ICD-10 diagnosis was significantly associated with oral psychopharmacological medications $(p<0.001)$, vitamins $(p<0.003)$, and injectable antipsychotics $(p<0.003)$ in our study setting.

The significant factors were further explored in detail, and it was found that males were predominantly referred from medicine, surgery, and orthopedics departments. Alcohol dependence was the most predominant ICD-10 diagnosis that emerged significantly across medicine and surgery departments, delirium was significant referral from the orthopedics department, and acute psychosis was from the ophthalmology department. Alcohol dependence was significantly found in males, while depression and adjustment disorders were significantly found in females. The use of vitamins and oral psychotropics was significant in patients with alcohol dependence.
The one-way ANOVA model was applied for exploring the relation between the number of days into psychiatric referral and referring unit; however, there was no statistically significant association as $F=1.583$ and significance was 0.161 . ANOVA also did not reveal any statistically significant association between the referring unit and the number of medical drugs as $F=1.823$ and significance was 0.103 (Table 2). One-way ANOVA for age and psychiatric diagnosis emerged significantly. Younger age was significantly found to be associated with deliberate self-harm and anxiety-related disorders. Middle and older age groups were associated with organic mental conditions, depression, schizophrenia, and substance use disorders.

The following variables did not emerge significantly in our study. The variable psychiatric diagnosis did not emerge significantly when explored with the residence $(p<0.308)$, vitamins prescribed $(p<0.15)$, injectable antipsychotics prescribed $(p<0.129)$, and communication about reference $(p<0.336)$. The variable ICD-10 disorder did not have any significant association with the residence $(p<0.551)$ and communication about reference $(p<0.206)$. The variable referring unit did not have significant associations with substance use $(p<0.635)$, residence $(p<0.295)$, dual diagnosis $(p<0.928)$, communication about reference $(p<0.836)$, reason for referral $(p<0.068)$, vitamins prescribed $(p<0.454)$, and medical diagnosis $(p<0.997)$.

\section{Discussion}

\section{Demographic Factors}

In our study, the mean age was 40.95 years with males being predominant. This was similar to the study done by Aghanva et al. in which the mean age was 31.1 (SD 13.1) years and the works of Risal and Sharma who found that the mean age of the subjects evaluated was $37.26( \pm 1.86)$ years. ${ }^{13,14}$ Keertish et al. found that the age distribution of the study population showed that a majority of the patients (59.6\%) belonged to the age group of $16-45$ years and a preponderance of males in both inpatients (58 vs 42\%). ${ }^{15}$ However, Aghanva et al. and Risal and Sharma found females contributing 
Patterns of Consultation-liaison Psychiatry among Inpatients at a Tertiary Hospital

Table 2: One-way ANOVA for age and regression with ICD-10 categories

\begin{tabular}{lllllll}
\hline & Sum of squares & DF & Mean square & $F$ & \multicolumn{2}{l}{ Sig. } \\
\hline Between groups & 6838.694 & 6 & 1139.782 & 4.036 & 0.001 & \\
Within groups & 26265.496 & 93 & 282.425 & & & \\
Total & 33104.190 & 99 & & & & \\
Age regressed with ICD-10 categories & $N$ & Mean & SD & Std. error & $95 \%$ confidence interval for mean \\
Organic mental disorders & 18 & 47.78 & 23.827 & 5.616 & 35.93 & 59.63 \\
Substance use disorders & 27 & 47.26 & 13.902 & 2.676 & 41.76 & 52.76 \\
Schizophrenia and other psychoses & 7 & 41.43 & 14.524 & 5.490 & 28.00 & 54.86 \\
Mood disorders & 17 & 42.94 & 18.972 & 4.601 & 33.19 & 52.70 \\
Anxiety and other stress-related disorders & 10 & 31.20 & 15.447 & 4.885 & 20.15 & 42.25 \\
Mental retardation & 3 & 25.33 & 12.702 & 7.333 & -6.22 & 56.89 \\
Deliberate self-harm & 18 & 27.61 & 11.099 & 2.616 & 22.09 & 33.13 \\
Total & 100 & 40.41 & 18.286 & 1.829 & 36.78 & 44.04 \\
\hline
\end{tabular}

DF is degree of freedom.

to 56 and $56.4 \%$, respectively. ${ }^{13,14}$ Ramdurg et al. found that $78 \%$ of the sample was from urban areas in their study, whereas in our study, the majority of the patients were hailing from rural areas. ${ }^{16}$

\section{Days of Referral to Psychiatry}

The mean days of referral to psychiatry as described by Arbabi et al. were 12.56 days ( range $=1-90, S D=13) .{ }^{17}$ In comparison, the mean day of referral to psychiatry in our study was relatively earlier (mean 2.27 days). Wong and Yiu found that $82 \%$ of patients referred for consultation were attended to within the same working day of receiving the referral and $99 \%$ were attended to by the end of the next working day. ${ }^{18}$

\section{Referring Departments}

Our study findings were similar to the findings of Ansari et al. and Rothenhausler et al. who also found that the medicine department contributed to maximum referrals to psychiatry $(74.4$ and $31 \%$, respectively). ${ }^{19,20}$ Sharp et al. found that the majority $(60.2 \%)$ of referrals in their study for consultation-liaison service came from physicians. ${ }^{21}$ Keertish et al. also found that the medicine department contributed to maximum referrals (59\%). ${ }^{15}$ Risal and Sharma also found that maximum (70.4\%) referral was from the medical ward.$^{14}$ However, Elisei et al. found that the majority of their referrals in their study settings were from the emergency room (51.4\%). They also found that medical wards contributed to $39 \%$ of referrals and surgical wards contributed to $9.4 \%$ of referrals. ${ }^{22}$ Barrimi et al. found that the dermatology (16\%) and nephrology (11\%) departments contributed to maximum referrals in their study. ${ }^{23}$ Wand found that the highest rate of referrals to psychiatry was from the emergency department, followed by obstetrics and gynecology departments. ${ }^{24}$

\section{Reasons for Referral to Psychiatry}

The top reason for referral to psychiatry for liaison consultation by Devasagayam and Clark was suicide evaluation, but this emerged as the second most common reason in our study as the most common reason for referral in our study was substance use. ${ }^{25}$ Sharp et al. found that depression (26.7\%) was the most common reason for referral. ${ }^{21}$ Anderson and Quante found that for more than $50 \%$ of the cases, their psychiatric illness was either directly or indirectly the reason for which they presented to the hospital. ${ }^{26}$ Wong and Yiu found that the top reasons for CLP were unstable emotions, followed by suicidal/ deliberate self-harm and aggression. ${ }^{18}$ Wand et al. found that the most common reason for referral was an assessment of risk. ${ }^{24} \mathrm{Fi} l e r$ and Quante found that the predominant reasons for referral to CLP services were assumed depressive symptoms, suicidal ideations, and "difficult" behavior. ${ }^{27}$ Keertish et al. found that the most common reasons for referrals were medically unexplained somatic complaints (23.1\%), anxiety (21\%), and abnormal behavior (13.1\%). ${ }^{15}$ Clarke and Smith found depression, suicide risk, and suspected psychological component to illness as the main reasons for referral to psychiatry. ${ }^{28}$

\section{Patterns of Psychiatric Disorders}

In our study, the most common psychiatric diagnosis was alcohol dependence (24\%). Aghanva et al. found depressive episode/ recurrent depressive disorder $(29.9 \%)$ as the most common psychiatric diagnosis in their study, whereas Clark and Smith found mood disorders (55\%) as the commonest diagnosis. ${ }^{13,28}$ Zuo et al. found organic brain syndromes, while Rothenhausler et al. found adjustment disorders as the most common psychiatric disorder across their both surveys $A$ and $B .^{20,29}$ Sharp et al. found that diagnoses of both mood (affective) disorders (32.9\%) and neurotic, stress-related, and somatoform disorders (54.7\%) were common in their study. ${ }^{21}$ Keertish et al. found that neurotic, stress-related, and somatoform disorders were the most common ones $(41.7 \%){ }^{15}$ Risal and Sharma found that depression was the most common diagnosis among patients referred from the medical ward $(34.7 \%)$ and anxiety was most commonly found among patients referred from emergency settings (24.5\%). ${ }^{14}$ Dual psychiatric diagnosis was found in $10 \%$ of our study patients. This is similar to the findings of Arbabi et al. who found that $10.9 \%$ of patients had received multiple psychiatric diagnoses. ${ }^{17}$

\section{Patterns of Pharmacotherapy and Psychotherapy}

Our rates of pharmacotherapy (58\%) for CLP patients were higher as compared to a study by Rothenhausler et al. (45-49\%) but lower than rates found by Barrimi et al. who found that pharmacological treatment was done in $60 \%$ of cases. ${ }^{20,23}$ Wand also found that the most common clinical management approach in CLP was pharmacotherapy. ${ }^{24}$

Sharp et al. found that pharmacological therapy was done in $25.4 \%$ of patients and cognitive behavior therapy $(56.1 \%)$ was the most commonly used intervention. ${ }^{21}$ We found that $42 \%$ of 
inpatients received only psychotherapy in our study. Gangat et al. found that psychotherapy as an intervention was offered to only $18 \%$ of the hospital patients referred for CLP. ${ }^{30}$

In our study, we noted that antipsychotics and benzodiazepines together were prescribed to $14 \%$ of patients and this was the most common pattern across pharmacotherapy. Schellhorn et al. found that geriatric patients were more likely to receive atypical antipsychotic medications. He also found that younger patients had received benzodiazepines but we did not find any significant association between age and pharmacotherapy. ${ }^{31}$ Anderson and Quante found that benzodiazepines emerged as the most commonly administered drugs for acute pharmacological intervention in their study. ${ }^{26}$

\section{Statistical Correlations}

In our study, the factors that emerged statistically significant with the referring unit were the ICD-10 diagnostic category $(p=0.001)$, psychiatric diagnosis ( $p=0.001)$, and gender (0.048). Gender also emerged statistically significant when regressed with a psychiatric diagnosis $(p=0.001)$. The ICD-10 disorder subtype category had a significant association with the use of vitamins ( $p=0.003)$, oral psychiatric medications (0.001), and injectable antipsychotics (0.003) in our study. Elisei et al. found statistically significant correlations between the source of referrals, the reasons for the referrals, psychiatric care prior to the evaluation, and the psychiatric disorder that was diagnosed during the assessment in their study. ${ }^{22}$ De Giorgio et al. found that significant associations emerged between gender and social status and occupation. They found that the clinical sector was related to the reason for referral, type of consultation, liaison investigations, and long-term treatment plan after hospital discharge. They also noted that the ICD-10 psychiatric diagnosis was associated with social and consultation-related factors ${ }^{32}$ Risal and Sharma found statistically significant correlation $(p<0.05)$ between the source of referral and psychiatric diagnosis. ${ }^{14}$

\section{Patient and Caregiver Communication Regarding Psychiatric Reference}

Seventy-four percent of patients as well as caregivers were not communicated adequately by the referring doctors regarding the reason for referral to psychiatry in our study. In a similar study assessing communication regarding psychiatric referrals, Bagheri et al. found that $68 \%$ of patients were not informed about a referral to psychiatry by their referring physicians. ${ }^{33}$ We speculate that stigma, misinformation, lack of training in communicating referrals, lack of knowledge toward psychiatric disorders, and attitudinal problems could be some of the reasons for poor communication to patients and caregivers. This observation needs further exploration, and we recommend future researchers to independently and systematically study this topic of communication regarding reference to psychiatry and factors influencing it.

\section{Rate of Referral to Psychiatry and Psychiatric Service Utilization}

In our study, the rate of referral was $0.36 \%$. Keertish et al. found that the referral rate was $0.42 \%$ and Gibson et al. found that the referral rate was $1.5 \% .{ }^{15,34}$ Wand et al. found that the rate of referral to CLP was $3 \% .{ }^{24}$ Windhager et al. found an increase in the pattern of referrals overall of $22 \%$ in a year with a multidisciplinary consulting team comprising of psychiatrists, psychologists, psychosomatic physicians, and social workers and was able to provide psychosocial consultation service at a quantitative level required by international guidelines. ${ }^{35}$
Zarghami et al. conducted a study on attitudes and practices of physicians regarding CLP and found that the most common reasons of physicians for not requesting psychiatric consultation were lack of time, forgetfulness, lack of access to psychiatrist, and lack of belief in the need for psychiatric consultation, respectively. ${ }^{36}$ However, Fißler and Quante suggest that CLP service was mostly "overprovided" and underutilized; hence, it is recommended that there was a need to develop precise guidelines for CLP services and relevant training in psychiatry for non-psychiatric physicians in order to achieve optimal holistic treatment and care for patients. ${ }^{27}$ Despite 30 years of development of CLP services across certain developed countries, several cultural and organizational problems in different countries have emerged regarding the implementation of CLP services and the application of CLP guidelines ${ }^{37}$ Kishi suggests that the time has come to reorient the focus on the improvement of the CLP service system through care coordination strategies. ${ }^{38}$ Specifically integrated serviced delivery models integrating care between primary care and general hospitals can be tremendously useful in reaching optimum utilization of consultation-liaison services. ${ }^{39}$ Lyne et al. recommend that the introduction of guidelines on CLP and their implementation can help in improving the quality of care services. ${ }^{40}$ Wood and Wand recommend that the future research in CLP can focus on exploring cost-effectiveness, length of stay, standardizing patient and staff feedback, and short-term patient outcomes with improved methodologies. ${ }^{41}$

\section{ConClusion}

We conclude that diverse patterns for CLP exist in our study and our study findings are relevant to our setting. The strength of our study was that it was based at a tertiary institution with adequate departments contributing to referrals as well as catering to a wide geographical area comprising of both rural and urban populations with diverse socioeconomic backgrounds. The study was conducted after the IEC approval with informed consent and appropriate statistics comprising of multiple logistic regressions and ANOVA. The limitations of our study were that we included adults and did not include children and adolescents. We analyzed the referring departments as per major specialties and did not include super specialty services in our study. This was another limitation of our study that it was a single-point study and there was no follow-up of patients conducted in our study.

Clinical Significance: We recommend that medical institutions should direct their attention toward assessing their patterns of psychiatric services for liaison psychiatry in order to improve the overall utilization of psychiatric liaison services. There may be a role to assess knowledge, perceptions, perceived stigma, and attitudes regarding liaison psychiatry across different units and departments at the institutional level to facilitate understanding regarding existing barriers so that interventions can be planned accordingly at the interdepartmental as well as at the institutional level to improve existing lacunae and improve psychiatric service utilization across CLP setting.

\section{References}

1. Lipsitt DR. Consultation-liaison psychiatry and psychosomatic medicine: the company they keep. Psychosom Med 2001;63(6):896909. DOI: 10.1097/00006842-200111000-00008.

2. Chen KY, Evans R, Larkins S. Why are hospital doctors not referring to Consultation-Liaison Psychiatry? - a systemic review. BMC Psychiatry 2016;9;16(1):390. DOI: 10.1186/s12888-016-1100-6. 
3. Carr VJ, Lewin TJ, Reid AL, et al. An evaluation of the effectiveness of a consultation-liaison psychiatry service in general practice. Aust N Z J Psychiatry 1997;31(5):714-725;discussion 726-7. DOI: 10.3109/00048679709062685.

4. Karasu TB, Plutchik R, Steinmuller RI, et al. Patterns of psychiatric consultation in a general hospital. Hosp Community Psychiatry 1977;28(4):291-294. DOI: 10.1176/ps.28.4.291.

5. Alhuthail YR. Timing of referral to consultation-liaison psychiatry. Int J Health Sci (Qassim) 2009;3(2):175-180.

6. Alaja R, Tienari P, Seppä K, et al. Patterns of comorbidity in relation to functioning (GAF) among general hospital psychiatric referrals. European Consultation-Liaison Workgroup. Acta Psychiatr Scand 1999;99(2):135-140. DOI: 10.1111/j.1600-0447.1999.tb07211.x.

7. Grover S, Avasthi A. Consultation-liaison psychiatry in India: where to go from here? Indian J Psychiatry 2019;61(2):117-124. DOI: 10.4103/ psychiatry.Indian J Psychiatry_255_17.

8. Grover S, Avasthi A. Consultation-liaison psychiatry services: a survey of medical institutes in India. Indian J Psychiatry 2018;60(3):300-306. DOI: 10.4103/psychiatry.Indian J Psychiatry_256_17.

9. Patra P, Divinakumar KJ, Prakash J, et al. Clinico-psycho-social profile of patients brought under consultation-liaison psychiatry care in a large tertiary care referral hospital. Ind Psychiatry J 2017;26(1):24-27. DOI: 10.4103/ipj.ipj_13_17.

10. Grover S, Sahoo S, Aggarwal S, et al. Reasons for referral and diagnostic concordance between physicians/surgeons and the consultation-liaison psychiatry team: an exploratory study from a tertiary care hospital in India. Indian J Psychiatry 2017;59(2):170-175. DOI: 10.4103/psychiatry.IndianJPsychiatry_305_16.

11. Hashim U, Kumar RS, Philip M. Consultation-liaison psychiatric service utilization by suicide attempters. Indian J Psychiatry. 2018;60(4): 427-432. DOI: 10.4103/psychiatry.IndianJPsychiatry_471_17.

12. Chaturvedi SK, Balaraju KB, Upadhyaya M, et al. Psychiatric referrals in a neuropsychiatric centre. Int J Soc Psychiatry 1989;35(2):197-203. DOI: $10.1177 / 002076408903500208$.

13. Aghanwa H. Consultation-liaison psychiatry in Fiji. Pac Health Dialog 2002;9(1):21-28.

14. Risal A, Sharma PP. Psychiatric morbidity patterns in referred inpatients of other specialties. JNMA J Nepal Med Assoc 2013;52(189): 238-244.

15. Keertish N, Sathyanarayana MT, Kumar BG, et al. Pattern of psychiatric referrals in a tertiary care teaching hospital in southern India. J Clin Diagn Res 2013;7(8):1689-1691. DOI: 10.7860/ JCDR/2013/6425.3238.

16. Ramdurg S, Goyal S, Goyal P, et al. Sociodemographic profile, clinical factors, and mode of attempt in suicide attempters in consultation liaison psychiatry in a tertiary care center. Ind Psychiatry J 2011;20(1):11-16. DOI: 10.4103/0972-6748.98408.

17. Arbabi M, Laghayeepoor R, Golestan B, et al. Diagnoses, requests and timing of 503 psychiatric consultations in two general hospitals. Acta Med Iran 2012;50(1):53-60.

18. Wong MM, Yiu MG. Consultation-liaison service in a regional hospital in Hong Kong. East Asian Arch Psychiatry 2014;24(2):51-57.

19. al-Ansari EA, el-Hilu S, el-Hihi MA, et al. Patterns of psychiatric consultations in Kuwait general hospital. Gen Hosp Psychiatry 1990;12(4):257-263. DOI: 10.1016/0163-8343(90)90064-j.

20. Rothenhäusler HB, Stepan A, Kreiner B, et al. Patterns of psychiatric consultation in an Austrian tertiary care center - results of a systematic analysis of 3,307 referrals over 2 years. Psychiatr Danub 2008;20(3):301-309.

21. Sharp J, Innes I, Brown T. Referrals to a liaison psychiatry outpatient clinic. Scott Med J 2011;56(3):125-129. DOI: 10.1258/smj.2011.011108.

22. Elisei S, Pauselli L, Balducci PM, et al. Mood disorders in general hospital inpatients: one-year data from a psychiatric consultationliaison service. Psychiatr Danub 2013;25(Suppl 2):S268-S271.
23. Barrimi M, Elghazouani F, Aarab C, et al. [Experience of liaison psychiatry in Morocco: transversal study over 24 months]. Encephale 2014;40(5):373-379. DOI: 10.1016/j.encep.2013.03.010.

24. Wand AP, Corr MJ, Eades SJ. Liaison psychiatry with Aboriginal and Torres Strait Islander peoples. Aust N Z J Psychiatry 2009;43(6): 509-517. DOI: 10.1080/00048670902873649.

25. Devasagayam D, Clarke D. Changes to inpatient consultation-liaison psychiatry service delivery over a 7-year period. Australas Psychiatry 2008;16(6):418-422. DOI: 10.1080/10398560802074676.

26. Anderson C, Quante A. Acute interventions and referral of patients with bipolar disorder by the psychiatric consultation liaison service in a general hospital in Germany: a retrospective analysis. Prim Care Companion CNS Disord 2014;16(2):PCC.13m01602. DOI: 10.4088/ PCC.13m01602.

27. Fißler M, Quante A. Psychiatric liaison consultations of patients without psychiatric illness in a general hospital in Germany: a retrospective analysis. Wien Med Wochenschr 2015;165(21-22):436444. DOI: 10.1007/s10354-015-0384-3.

28. Clarke DM, Smith GC. Consultation-liaison psychiatry in general medical units. Aust N Z J Psychiatry 1995;29(3):424-432. DOI: 10.3109/00048679509064950.

29. Zuo C, Yang L, Chu CC. Patterns of psychiatric consultation in a Chinese general hospital. Am J Psychiatry 1985;142(9):1092-1094. DOI: 10.1176/ajp.142.9.1092.

30. Gangat AE, Naidoo LR, Simpson MA. Referral patterns for psychiatric consultation in a large general hospital. S Afr Med J 1987;72(12): 853-855.

31. Schellhorn SE, Barnhill JW, Raiteri V, et al. A comparison of psychiatric consultation between geriatric and non-geriatric medical inpatients. Int J Geriatr Psychiatry 2009;24(10):1054-1061. DOI: 10.1002/gps.2221.

32. De Giorgio G, Quartesan R, Sciarma T, et al. Consultation-Liaison Psychiatry - from theory to clinical practice: an observational study in a general hospital. BMC Res Notes 2015;8:475. DOI: 10.1186/s13104015-1375-6.

33. Bagheri AS, Lane LS, Kline FM, et al. Why physicians fail to tell patients a psychiatrist is coming. Psychosomatics 1981;22(5):407-419. DOI: 10.1016/S0033-3182(81)73510-2.

34. Gibson RC, Martin JS, Neita SM. Mental illness and public health: exploring the role of general hospital physicians at a teaching hospital in Jamaica. West Indian Med J 2010;59(6):662-667.

35. Windhager E, Thaler K, Selberis-Vahl WV, et al. [Feasibility of consultation-liaison psychiatry in a large general hospital: quantitative description of services and personnel expenditure]. Neuropsychiatr 2015;29(2):84-87. DOI: 10.1007/s40211-015-0146-x.

36. Zarghami M, Farnia S, Khalilian AR, et al. Study of attitudes and practice of physicians regarding consultation-liaison psychiatry in teaching hospitals of Mazandaran, Iran. Iran J Psychiatry Behav Sci 2014;8(2):38-43.

37. Grassi L, Mitchell AJ, Otani M, et al. Consultation-liaison psychiatry in the general hospital: the experience of UK, Italy, and Japan. Curr Psychiatry Rep 2015;17(6):44. DOI: 10.1007/s11920-015-0581-1.

38. Kishi Y. [The current state and further development of consultationliaison psychiatry in general hospitals]. Seishin Shinkeigaku Zasshi 2010;112(12):1203-1209.

39. Kirchen-Peters S, Diefenbacher A. [Geropsychiatric consultationliaison services. An answer to the challenges of dementia?].Z Gerontol Geriatr 2014;47(7):595-604. DOI: 10.1007/s00391-013-0561-1.

40. Lyne J, O'Donoghue B, Bonnar M, et al. Guidelines reduce time to consultation for a liaison psychiatry service in an Irish teaching hospital. Ir J Med Sci 2012;181(2):185-188. DOI: 10.1007/s11845-0100511-5.

41. Wood R, Wand AP. The effectiveness of consultation-liaison psychiatry in the general hospital setting: a systematic review. J Psychosom Res 2014;76(3):175-192. DOI: 10.1016/j.jpsychores.2014.01.002. 